\title{
What do we know about optimal nutritional strategies in children with pediatric acute respiratory distress syndrome?
}

\author{
Rajalakshmi Iyer, Arun Bansal \\ Department of Pediatrics, Advanced Pediatrics Centre, Postgraduate Institute of Medical Education and Research (PGIMER), Chandigarh, India \\ Contributions: (I) Conception and design: A Bansal; (II) Administrative support: A Bansal; (III) Provision of study materials or patients: All authors; \\ (IV) Collection and assembly of data: None; (V) Data analysis and interpretation: None; (VI) Manuscript writing: All authors; (VII) Final approval of \\ manuscript: All authors. \\ Correspondence to: Arun Bansal. Professor, Department of Pediatrics, Advanced Pediatrics Centre, Postgraduate Institute of Medical Education and \\ Research (PGIMER), Chandigarh 160012, India. Email: drarunbansal@gmail.com.
}

\begin{abstract}
Nutrition in pediatric acute respiratory distress syndrome (PARDS) is an essential aspect of therapy, with potential to modify outcomes. The gut is slowly establishing its place as the motor of critical illness, and the 'gut-lung' axis has been shown to be in play in the systemic inflammatory response. Thus, utilizing the gut to modify outcomes in PARDS is an exciting prospect. PARDS is associated with high mortality in low- and middle-income countries (LMIC), where malnutrition is also prevalent and may worsen during hospital stay. Mortality may be higher in this subgroup of patients. At present, the gold standard to estimate resting energy expenditure (REE) in critically ill children is indirect calorimetry. However, it is a cumbersome and expensive procedure, as a result of which its routine practice is limited to very few units across the world. Therefore, predictive equations, which may under- or over-estimate REE, are relied upon to approximate calorie and protein needs of children with PARDS. Despite having target calorie and protein requirements, studies have found that a large proportion of critically ill children do not achieve these levels even at the end of a week in pediatric intensive care unit (PICU). The preferred mode of nutrition delivery is enteral, and if possible, early enteral nutrition (EEN). Immunonutrition has been a lucrative subject of research, and while there have been some strides, no therapy has yet conclusively demonstrated benefit in terms of mortality or reduced length of stay in PICU or the hospital. Probable immunonutrients in PARDS include omega-3 fatty acids, arginine, glutamine and vitamin D, though none are a part of any recommendations yet.
\end{abstract}

Keywords: Pediatric acute respiratory distress syndrome (PARDS); critically ill children; PALICC; nutrition; indirect calorimetry

Submitted Apr 23, 2019. Accepted for publication Aug 01, 2019.

doi: 10.21037/atm.2019.08.25

View this article at: http://dx.doi.org/10.21037/atm.2019.08.25

\section{Introduction}

The child with acute respiratory distress syndrome (ARDS) presents a challenge to pediatric intensivists, with high severity of illness and multi-organ failure. Thus, the focus of the PICU is always towards stabilization, optimization of mechanical ventilation and meeting targets related to oxygenation.

In the midst of life support measures, an area often pushed to the background is nutrition. Evidence and literature over time have demonstrated the importance of the part played by nutrition in the critically ill. The recent Pediatric Acute Lung Injury Consensus Conference (PALICC) guidelines have also stressed the importance of nutrition in the child with pediatric acute respiratory distress syndrome (PARDS), although there is not much robust data on the same (1).

Moderate to severe PARDS has a mortality to the tune 
of $25-35 \%$ in the developed world (2). This figure is higher in low and middle income countries, going to as high as $45-60 \%(3-5)$. It is of paramount importance, therefore, to find ancillary therapies that may reduce mortality beyond the reduction that has been achieved by application of low tidal volume and high PEEP. Nutrition is one of the cornerstones of PICU practice. While it may take a backseat in the face of resuscitative and technologically advanced therapy, its importance cannot be understated. If nutritional intervention, as simplistic as it may seem, can play a role in reduction of morbidity and mortality, an understanding of its nuances in ARDS is key to its implementation. This review highlights these key concepts.

\section{Gut as a motor of critical illness}

The theory that the gut is the motor of critical illness has been a subject of study for the last few decades. The gut is the largest mucosal surface that is in contact with the outside world (6). It is lined by a single layer of epithelial cells, and has its own lymphoid tissue and microbiome; it is prone to disruption, bacterial translocation and a strong immune response. The integrity of the gut may be altered at every level-the mucus layer, the epithelial layer, the submucosal layer and reduced renewal of cells in the epithelium. Experimental data in mice have reinforced the theory of bacterial translocation, which was the older concept of why gut injury could lead to manifestations of distant injury, especially in lungs (7). This damage is contributed by the bacteria, endotoxins and cytokine response in the gut (8). It has also been found in animal models, that bacterial virulence may be modified by the host environment- if commensal bacteria find themselves in an environment that may be beneficial to them, they undergo a virulent change and may cause severe disease in the host. This applies to sepsis, septic shock, and major surgery (9). However, the same results have not been replicated in human beings. Therefore, the present theory is not limited to only bacterial translocation. The gut has extensive lymphoid tissue and cytokines produced in the gut are carried via mesenteric lymph to the thoracic duct and then the lungs. Thus, gut injury may perpetuate lung injury. A study of probiotics in critically ill children with severe sepsis found that pro-inflammatory cytokine levels were significantly reduced, while anti-inflammatory cytokines were elevated, in those who were administered probiotics (10). Whether this has therapeutic implication or not is unknown, but it reinforces the theory that the gut plays a definitive role in systemic inflammation (10). Each of these processes is believed to augment distant organ injury (8,9,11-14).

\section{The gut-lung axis}

The critically ill child with, for example, PARDS or septic shock, has a number of organ injuries at presentation or during evolution of the illness. The presence of shock, multiple vasoactive drug infusions and hypoxia predispose the gut to injury, thereby leading to the above mentioned adverse consequences. The lung and the gut have mucosa in continuum, but with different microbiomes. The normal, non-diseased alveoli, are populated with nonpathogenic anaerobic bacteria like Prevotella, Veillonella, and Fusobacterium, while the normal gut flora include the Bacteroidetes and Enterobacteriaceae species (11). It has been seen that in the diseased states mentioned above, there occurs a state of dysbiosis of the lung, with bacteria which normally populate the gut, being found in the lung. This may also occur due to continuous micro-aspiration of oropharyngeal secretions from around the endotracheal tube in an intubated patient (11,15-18). Hence, it seems prudent to consider the various effects the gut may play on lung injury in a critically ill child and attempt measures to keep the gut as healthy as possible.

\section{Nutrition in PARDS}

Experience and some evidence, which isn't very robust, have reiterated the importance of nutrition in the child with ARDS. This area has always raised more questions than answers and most practice is consensus based, rather than on hard evidence. What is well known, however, are the effects of malnutrition on outcomes and the malnourishment that critically ill children are prone to in PICU stay. Malnutrition is either present at admission, or may develop or worsen in more than half the children admitted to intensive care units (19-21). Studies have also shown that malnutrition is an independent risk factor for longer duration of mechanical ventilation and acquisition of health care associated infections $(22,23)$. In addition, malnutrition has an impact on mortality. In an observational study on PARDS by Yadav et al., $63 \%$ of malnourished children died (17 children out of 27 malnourished children with PARDS) (5). A recent retrospective study by Wong et al., in 107 children with ARDS showed that those children who received adequate calories had lesser mortality (34.6\% vs. $60.5 \%, \mathrm{P}=0.025)$, while those who received adequate 
protein had both, reduction in mortality (14.3\% vs. $60.2 \%$, $\mathrm{P}=0.002$ ) and more VFDs [median (interquartile range), 12 (3.0-19.0) vs. $0(0.0-14.8)$ days; $\mathrm{P}=0.005]$ (24). Hence, it is quite clear that nutrition in PARDS is an area which needs more careful thought and planning in all children admitted to PICUs, and may aid in improvement in outcomes like length of mechanical ventilation, PICU stay and mortality.

\section{Enteral vs. parenteral nutrition}

Critically ill children are at risk of gut barrier disruption and splanchnic vascular compromise, due to the presence of shock, vasoactive support and hypoxia (25). In addition, there are physician-related barriers for nutrition, like the need for multiple procedures, like intubation, central venous catheter insertion, and emergent surgery, all of which require the patient to be in a 'nil-per-oral' status $(26,27)$. Hence, both the initiation of nutrition, and the debate of enteral versus parenteral nutrition has been the subject of critical care controversies for many decades now.

\section{Why enteral nutrition $(E N)$ ?}

$\mathrm{EN}$ is the natural mode of feeding and seems to inherently have its advantages. Animal studies have shown that enteral feeds "keep the gut moving", thereby reducing bacterial translocation, prevent mucosal atrophy, maintain integrity of the epithelial barrier of the gut and reduce production of toxic cytokines (28). The exact opposite effects have also been demonstrated in murine models using total parenteral nutrition (TPN) (29,30). Older studies have also shown higher incidence of new onset sepsis and hyperglycemia in those who were on TPN (31). It seems logical, therefore, to 'use the gut' to feed the sick child, as soon as possible, and prefer EN over TPN.

\section{When should it be started?}

The question of how soon is also something which has been the subject of investigation. Studies have compared early enteral nutrition (EEN) to late enteral nutrition, defining EEN as within 48-72 hours of admission to the PICU. EEN was associated with shorter PICU and overall length of stay, and reduction in mortality $(19,32,33)$. Therefore, once the child has attained hemodynamic stability and there are no other physician-decided barriers to feeding, EN, and preferably EEN should be initiated.

Although one may be enthusiastic about initiating $\mathrm{EN}$, it has been observed that hardly $40-75 \%$ of total goal calories and proteins are met in the first 7 days of PICU stay in the critically ill child $(34,35)$. There are many barriers to EN, as have already been mentioned above. In addition, one of chief factors operating in the inability to meet caloric and protein targets is feed interruptions, due to actual or perceived gastric intolerance and for procedures. A study done by Mehta et al. found that almost $50 \%$ of all feed interruptions are avoidable (36). The presence of a written feeding protocol, with guidelines on feed intolerance would likely reduce the number of interruptions and aid in achieving full feeds as early as possible.

\section{How much to feed?}

The ideal method to decide how much to feed a child is dependent on the resting energy expenditure (REE), which is the requirement to sustain the basal metabolic rate. This may be calculated using mathematical equations like the Schofield equation or FAO/WHO/UN equations, or by the gold standard method, indirect calorimetry. Predictive equations are inaccurate and may overestimate the needs of the child (37-39). Indirect calorimetry is cumbersome and is not available in most centres for routine use, except for academic purposes. A recent study done by Ismail et al., assessing energy balance in critically ill mechanically ventilated children, showed poor agreement between energy expenditure calculated by predictive equations and indirect calorimetry (40). But for practical purposes, either of the equations mentioned may be used for calculation of calorie requirement (41).

Both, underfeeding and overfeeding are deleterious. Underfeeding leads to endogenous protein breakdown, loss of muscle mass and weakness of the muscles of respiration (42). This is associated with delay in weaning from ventilatory support, immunosuppression, delayed wound healing and increased risk of nosocomial infections. Overfeeding, on the other hand, is associated with higher carbon dioxide $\left(\mathrm{CO}_{2}\right)$ production, which may also lead to delay in weaning from ventilatory support (42). Another theory, which supports a degree of underfeeding (called permissive underfeeding), is autophagy. It is an evolutionarily conserved mechanism of clearing intracytoplasmic debris, and in the process, providing nutritive substrate (amino acids) to the cell. Autophagy is induced by starvation and oxidative stress, as seen in mild critical illness. Factors such as endotoxins, oxidative stress, ischemia, and mitochondrial dysfunction stimulate 
autophagy, which maintains ATP production, removes damaged proteins and improves cell survival. However, with increasing severity of illness, the same stimulatory factors lead to excessive autophagy, greater degradation of cytosolic proteins and organelles, and increased cell death. When and where this balance is tilted is unknown, but the benefits of permissive under-feeding may be due to autophagy (43-45).

In PARDS, the child is in a hyper-catabolic state, with higher than usual requirement of proteins and calories $(41,42)$. The breakdown of lean muscle and protein turnover may not completely be stopped by provision of adequate calories and proteins, but is markedly reduced. As per the latest ASPEN guidelines, a minimum protein intake of $1.5 \mathrm{~g} / \mathrm{kg} /$ day is advised in critically ill children (41).

Calories are met by providing both, carbohydrates and lipids. There has been some research into which type of feeding would be best suited to patients with ARDS. Traditional feeds contain 40-50\% carbohydrates and less than $30 \%$ as lipids. However, there have been postulations that high lipid, low carbohydrate formulations may be more beneficial. Some studies in adults have shown that a higher lipid formulation is associated with lower $\mathrm{CO}_{2}$ production as compared to higher carbohydrate formulations, resulting in lower ventilation and ICU days (46). This theory was later refuted, when Talpers et al. demonstrated in their study that it was increasing total calories, and not the carbohydrate content of the feed, which was responsible for higher $\mathrm{CO}_{2}$ production. They showed that $\mathrm{CO}_{2}$ production increased significantly, even when the carbohydrate: lipid ratio was maintained a constant, as total calories increased (47).

At present, guidelines suggest using balanced ratios of carbohydrates and lipids (41).

\section{Immunonutrition in PARDS}

\section{$\omega-3$ Fatty Acids and GLA}

The rationale for using eicosapentaenoic acid (EPA) and its related molecules in PARDS is that it may downregulate the production of inflammatory leukotrienes, thereby reducing inflammation, and may enhance production of positive modulators of inflammation. Animal models of sepsis-induced ARDS had shown that low-carbohydrate, high-fat diet containing EPA (fish oil), gamma-linolenic acid (GLA; borage oil) (EPA + GLA), and antioxidants improves lung microvascular permeability, oxygenation, and cardiopulmonary function and reduces pro-inflammatory eicosanoid synthesis and lung inflammation (46). Based on this, Gadek et al. undertook a study in adults with ARDS, with the treatment group receiving EPA + GLA diet, and controls receiving routine diets. They demonstrated a significant improvement in $\mathrm{PaO}_{2} / \mathrm{FiO}$ ratio on days 4 and 7 , reduction in BAL neutrophils by almost 2.5 -fold, and reduction in ventilation days and ICU length of stay in the treatment group versus controls (48).

A meta-analysis assessed 3 studies in adults with acute lung injury, who received EPA + GLA vs. control diet. A total of 296 patients were assessed, of whom 152 received $\mathrm{EPA}+\mathrm{GLA}$ and 144 were in the control group. There was significant reduction in mortality risk [OR $0.40 ; 95 \%$ confidence interval (CI), 0.24-0.68; $\mathrm{P}=0.001$ ], duration of mechanical ventilation [standardized mean difference $(\mathrm{SMD})=0.56 ; 95 \% \mathrm{CI}, 0.32-0.79 ; \mathrm{P}<0.0001]$, risk of developing new organ failure (OR 0.17; 95\% CI, 0.08-0.34; $\mathrm{P}<0.0001$ ) and in length of ICU stay (SMD $=0.51 ; 95 \%$ CI, 0.27-0.74; $\mathrm{P}<0.0001$ ), in those who were in the treatment group (49).

There was concern about diarrhea and feed intolerance following administration of these lipid formulations. However, there was no intractable diarrhea requiring stoppage of the feeds in any of the studies analysed (50).

The EDEN-omega study was conducted as part of the ARDSnet trials (Early Versus Delayed Enteral Feeding and Omega-3 Fatty Acid/Antioxidant Supplementation for Treating People With Acute Lung Injury or Acute Respiratory Distress Syndrome) (51). In addition to a comparison of feeding patterns in adults with ARDS, it also studied a combination of omega- 3 fatty acids and antioxidants, and placebo. In contrast to the other 3 major studies done on EPA + GLA, which used continuous enteral infusions of the lipid formulation, the EDEN-omega study used 12-hourly boluses of the lipids. The study, however, was terminated after interim analysis, for futility. The mortality at 60 days was significantly lower at in the control group versus the experimental cohort (16.3\% vs. 26.6\%; $\mathrm{P}=0.05)$. The control group also had more ventilator-free and ICU-free days. The final results of this study are yet to be published (42).

The first study on critically ill children using EPA + GLA showed a favourable modification of the fatty acid profile, but did not assess changes in oxygenation indices, ventilation days, length of PICU or hospital stay, or mortality (52). Another similarly timed trial in children using $\omega$-3 fatty acids and glutamine also showed a favourable lipid profile, but no difference in end points of ventilation days or mortality (53).

At present, there is no evidence for routine administration 
of $\omega-3$ Fatty Acids in PARDS and further studies are needed in children before a recommendation can be made (1).

\section{Arginine}

Arginine is a non-essential amino acid in normal states, as small quantities of it are produced by the body. However, in states of stress, it becomes an essential amine acid, which plays an important role in detoxification of ammonia, nitric oxide release, wound healing, and release of anabolic hormones. Hence, it has garnered interest as a potential immunonutrient (54).

A study done by van Waardenburg et al. on plasma concentration of arginine in critically ill children showed that levels were low in the acute phase, and a strong inverse relationship with inflammatory markers like CRP (55). Studies using arginine supplementation in adults have been controversial, with some beneficial effects in surgical patients, but with a trend towards increased mortality in septic patients (56). In the few studies done in children, arginine appears to have some immunomodulatory effects, but whether these translate into benefit in hard outcomes is yet to be seen $(57,58)$.

Further studies are required in PARDS for the role of arginine supplementation.

\section{Glutamine}

Glutamine is the most abundant amino acid in the body and similar to the lines of arginine, due to its high requirement in critical illness, it is a non-essential amino acid, which may become essential in these states. It has also attracted a lot of attention as a possible immunonutrient.

Studies in mice induced with both pulmonary and extrapulmonary ARDS have shown mitigation of lung injury, improvement in inflammatory markers and reduced systemic inflammation (59). There are, however, no studies in adults or children with lung injury which reiterate these findings.

\section{Vitamin D}

Vitamin D deficiency has associated with impaired pulmonary function and increased predilection for both, viral and bacterial infections, and non-infectious diseases of the lung like asthma. Vitamin D is believed to be an immunomodulatory, playing an important role in the Th2 response, macrophage, lymphocyte and epithelial cell function (60). Therefore, it seems logical to postulate that it may benefit in ARDS (61). A study in critically ill children showed that sepsis was associated with low vitamin D levels, as compared to matched controls, but there was no statistically significant difference in mortality, length of PICU stay or mechanical ventilation (62).

\section{Otber therapies}

Other nutrients that have been studied in the critically ill are zinc, selenium and anti-oxidants like ginger extract. Most studies have been conducted in adults, and have shown some benefit in reducing inflammation, but none are conclusive (41,58,63-65).

\section{Summary}

Nutritional therapy may play a pivotal role in PARDS and is as essential as other treatment modalities. EN, as early as feasible, should be considered. The ideal method of calculating REE is indirect calorimetry, but in its absence, predictive equations may be used (Schofield or FAO/ WHO/UN equations). Protein delivery should target at least $1.5 \mathrm{~g} / \mathrm{kg} /$ day. Both under- and over-feeding may be deleterious, and care must be taken to prevent malnutrition from occurring during PICU stay, as it is associated with serious adverse outcomes. Immunonutrients are a subject of research at present, and while some data in adults seem favourable towards the use of omega-3 fatty acids and borage oils (EPA + GLA), there is inconclusive data in children at present. Current guidelines do not recommend the use of immunonutrition in PARDS, but further studies are required.

\section{Acknowledgments}

None.

\section{Footnote}

Conflicts of Interest: The authors have no conflicts of interest to declare.

Ethical Statement: The authors are accountable for all aspects of the work in ensuring that questions related to the accuracy or integrity of any part of the work are appropriately investigated and resolved. 


\section{References}

1. Valentine SL, Nadkarni VM, Curley MA, et al. Nonpulmonary treatments for pediatric acute respiratory distress syndrome: proceedings from the Pediatric Acute Lung Injury Consensus Conference. Pediatr Crit Care Med 2015;16:S73-85.

2. Erickson S, Schibler A, Numa A, et al. Acute lung injury in pediatric intensive care in Australia and New Zealand: a prospective, multicenter, observational study. Pediatr Crit Care Med 2007;8:317-23.

3. Gupta S, Sankar J, Lodha R, et al. Comparison of Prevalence and Outcomes of Pediatric Acute Respiratory Distress Syndrome Using Pediatric Acute Lung Injury Consensus Conference Criteria and Berlin Definition. Front Pediatr 2018;6:93.

4. Lalgudi Ganesan S, Jayashree M, Chandra Singhi S, et al. Airway Pressure Release Ventilation in Pediatric Acute Respiratory Distress Syndrome. A Randomized Controlled Trial. Am J Respir Crit Care Med 2018;198:1199-207.

5. Yadav B, Bansal A, Jayashree M. Clinical Profile and Predictors of Outcome of Pediatric Acute Respiratory Distress Syndrome in a PICU: A Prospective Observational Study. Pediatr Crit Care Med 2019. Available online: https://journals.lww.com/pccmjournal/ Abstract/onlinefirst/Clinical_Profile_and_Predictors_of_ Outcome_of.98276.aspx

6. Klingensmith NJ, Coopersmith CM. The Gut as the Motor of Multiple Organ Dysfunction in Critical Illness. Crit Care Clin 2016;32:203-12.

7. Dickson RP, Erb-Downward JR, Huffnagle GB. The role of the bacterial microbiome in lung disease. Expert Rev Respir Med 2013;7:245-57.

8. Coopersmith CM, Stromberg PE, Davis CG, et al. Sepsis from Pseudomonas aeruginosa pneumonia decreases intestinal proliferation and induces gut epithelial cell cycle arrest. Crit Care Med 2003;31:1630-7.

9. Badami CD, Senthil M, Caputo FJ, et al. Mesenteric lymph duct ligation improves survival in a lethal shock model. Shock 2008;30:680-5.

10. Angurana SK, Bansal A, Singhi S, et al. Evaluation of Effect of Probiotics on Cytokine Levels in Critically Ill Children With Severe Sepsis: A Double-Blind, PlaceboControlled Trial. Crit Care Med 2018;46:1656-64.

11. Clark JA, Coopersmith CM. Intestinal crosstalk: a new paradigm for understanding the gut as the "motor" of critical illness. Shock 2007;28:384-93.

12. Mittal R, Coopersmith CM. Redefining the gut as the motor of critical illness. Trends Mol Med 2014;20:214-23.

13. Perrone EE, Jung E, Breed E, et al. Mechanisms of methicillin-resistant Staphylococcus aureus pneumoniainduced intestinal epithelial apoptosis. Shock 2012;38:68-75.

14. Neal MD, Richardson WM, Sodhi CP, et al. Intestinal Stem Cells and their Roles during Mucosal Injury and Repair. J Surg Res 2011;167:1-8.

15. Akrami K, Sweeney DA. The microbiome of the critically ill patient. Curr Opin Crit Care 2018;24:49-54.

16. Kelly BJ, Imai I, Bittinger K, et al. Composition and dynamics of the respiratory tract microbiome in intubated patients. Microbiome 2016;4:7.

17. Marsland BJ, Trompette A, Gollwitzer ES. The Gut-Lung Axis in Respiratory Disease. Ann Am Thorac Soc 2015;12 Suppl 2:S150-6.

18. Mukherjee S, Hanidziar D. More of the Gut in the Lung: How Two Microbiomes Meet in ARDS. Yale J Biol Med 2018;91:143-9.

19. Briassoulis G, Zavras N, Hatzis T. Malnutrition, nutritional indices, and early enteral feeding in critically ill children. Nutrition 2001;17:548-57.

20. Mehta NM, Duggan CP. Nutritional deficiencies during critical illness. Pediatr Clin North Am 2009;56:1143-60.

21. Delgado AF, Okay TS, Leone C, et al. Hospital malnutrition and inflammatory response in critically ill children and adolescents admitted to a tertiary intensive care unit. Clinics (Sao Paulo) 2008;63:357-62.

22. de Souza Menezes F, Leite HP, Koch Nogueira PC. Malnutrition as an independent predictor of clinical outcome in critically ill children. Nutrition 2012;28:267-70.

23. de Mello MJ, de Albuquerque M de FPM, Ximenes RA de A, et al. Factors associated with time to acquisition of bloodstream infection in a pediatric intensive care unit. Infect Control Hosp Epidemiol 2010;31:249-55.

24. Wong JJ, Han WM, Sultana R, et al. Nutrition Delivery Affects Outcomes in Pediatric Acute Respiratory Distress Syndrome. JPEN J Parenter Enteral Nutr 2017;41:1007-13.

25. Wells DL. Provision of enteral nutrition during vasopressor therapy for hemodynamic instability: an evidence-based review. Nutr Clin Pract 2012;27:521-6.

26. Rogers EJ, Gilbertson HR, Heine RG, et al. Barriers to adequate nutrition in critically ill children. Nutrition 2003;19:865-8.

27. Lee H, Koh SO, Kim H, et al. Avoidable Causes of Delayed Enteral Nutrition in Critically Ill Children. J 
Korean Med Sci 2013;28:1055-9.

28. Fukatsu K, Zarzaur BL, Johnson CD, et al. Enteral Nutrition Prevents Remote Organ Injury and Death After a Gut Ischemic Insult. Ann Surg 2001;233:660-8.

29. Buchman AL, Moukarzel AA, Bhuta S, et al. Parenteral nutrition is associated with intestinal morphologic and functional changes in humans. JPEN J Parenter Enteral Nutr 1995;19:453-60.

30. Yang H, Feng Y, Sun X, et al. Enteral versus Parenteral Nutrition: Effect on Intestinal Barrier Function. Ann N Y Acad Sci 2009; 1165:338.

31. MacFie J. Enteral versus parenteral nutrition: the significance of bacterial translocation and gut-barrier function. Nutrition 2000;16:606-11.

32. Haney A, Burritt E, Babbitt CJ. The impact of early enteral nutrition on pediatric acute respiratory failure. Clin Nutr ESPEN 2018;26:42-6.

33. Khalid I, Doshi P, DiGiovine B. Early enteral nutrition and outcomes of critically ill patients treated with vasopressors and mechanical ventilation. Am J Crit Care 2010;19:261-8.

34. de Neef M, Geukers VG, Dral A, et al. Nutritional goals, prescription and delivery in a pediatric intensive care unit. Clin Nutr 2008;27:65-71.

35. Malakouti A, Sookplung P, Siriussawakul A, et al. Nutrition support and deficiencies in children with severe traumatic brain injury. Pediatr Crit Care Med 2012;13:e18-24.

36. Mehta NM, McAleer D, Hamilton S, et al. Challenges to optimal enteral nutrition in a multidisciplinary pediatric intensive care unit. JPEN J Parenter Enteral Nutr 2010;34:38-45.

37. Hardy CM, Dwyer J, Snelling LK, et al. Pitfalls in predicting resting energy requirements in critically ill children: a comparison of predictive methods to indirect calorimetry. Nutr Clin Pract 2002;17:182-9.

38. Havalad S, Quaid MA, Sapiega V. Energy expenditure in children with severe head injury: lack of agreement between measured and estimated energy expenditure. Nutr Clin Pract 2006;21:175-81.

39. Mehta NM, Bechard LJ, Dolan M, et al. Energy imbalance and the risk of overfeeding in critically ill children. Pediatr Crit Care Med 2011;12:398-405.

40. Ismail J, Bansal A, Jayashree M, et al. Energy Balance in Critically Ill Children With Severe Sepsis Using Indirect Calorimetry: A Prospective Cohort Study. J Pediatr Gastroenterol Nutr 2019;68:868-73.

41. Mehta NM, Skillman HE, Irving SY, et al. Guidelines for the Provision and Assessment of Nutrition Support Therapy in the Pediatric Critically Ill Patient: Society of Critical Care Medicine and American Society for Parenteral and Enteral Nutrition. JPEN J Parenter Enteral Nutr 2017;41:706-42.

42. Krzak A, Pleva M, Napolitano LM. Nutrition therapy for ALI and ARDS. Crit Care Clin 2011;27:647-59.

43. McClave SA, Weijs PJ. Preservation of autophagy should not direct nutritional therapy. Curr Opin Clin Nutr Metab Care 2015;18:155-61.

44. Rosenthal MD, Carrott P, Moore FA. Autophagy: should it play a role in ICU management? Curr Opin Crit Care 2018;24:112-7.

45. Van Dyck L, Casaer MP, Gunst J. Autophagy and Its Implications Against Early Full Nutrition Support in Critical Illness. Nutr Clin Pract 2018;33:339-47.

46. al-Saady NM, Blackmore CM, Bennett ED. High fat, low carbohydrate, enteral feeding lowers $\mathrm{PaCO} 2$ and reduces the period of ventilation in artificially ventilated patients. Intensive Care Med 1989;15:290-5.

47. Talpers SS, Romberger DJ, Bunce SB, et al. Nutritionally associated increased carbon dioxide production. Excess total calories vs high proportion of carbohydrate calories. Chest 1992;102:551-5.

48. Gadek JE, DeMichele SJ, Karlstad MD, et al. Effect of enteral feeding with eicosapentaenoic acid, gammalinolenic acid, and antioxidants in patients with acute respiratory distress syndrome. Enteral Nutrition in ARDS Study Group. Crit Care Med 1999;27:1409-20.

49. Pontes-Arruda A, Demichele S, Seth A, et al. The use of an inflammation-modulating diet in patients with acute lung injury or acute respiratory distress syndrome: a metaanalysis of outcome data. JPEN J Parenter Enteral Nutr 2008;32:596-605.

50. Ferrie S. Re: The use of an inflammation-modulating diet in patients with acute lung injury or acute respiratory distress syndrome: a meta-analysis of outcome data. JPEN J Parenter Enteral Nutr 2010;34:452; author reply 453-4.

51. Early Versus Delayed Enteral Feeding and Omega-3 Fatty Acid/Antioxidant Supplementation for Treating People With Acute Lung Injury or Acute Respiratory Distress Syndrome (The EDEN-Omega Study) - Full Text View - ClinicalTrials.gov [Internet]. Available online: https:// clinicaltrials.gov/ct2/show/NCT00609180

52. Jacobs BR, Nadkarni V, Goldstein B, et al. Nutritional immunomodulation in critically ill children with acute lung injury: feasibility and impact on circulating biomarkers. Pediatr Crit Care Med 2013;14:e45-56.

53. Briassoulis G, Filippou O, Hatzi E, et al. Early enteral administration of immunonutrition in critically ill children: 
results of a blinded randomized controlled clinical trial. Nutrition 2005;21:799-807.

54. Morris SM. Arginine: beyond protein. Am J Clin Nutr 2006;83:508S-12S.

55. van Waardenburg DA, de Betue CT, Luiking YC, et al. Plasma arginine and citrulline concentrations in critically ill children: strong relation with inflammation. Am J Clin Nutr 2007;86:1438-44.

56. Zhou M, Martindale RG. Arginine in the critical care setting. J Nutr 2007;137:1687S-92S.

57. Marin VB, Rodriguez-Osiac L, Schlessinger L, et al. Controlled study of enteral arginine supplementation in burned children: impact on immunologic and metabolic status. Nutrition 2006;22:705-12.

58. Levy J. Immunonutrition: the pediatric experience. Nutrition 1998;14:641-7.

59. Oliveira GP, de Abreu MG, Pelosi P, et al. Exogenous Glutamine in Respiratory Diseases: Myth or Reality?

Cite this article as: Iyer R, Bansal A. What do we know about optimal nutritional strategies in children with pediatric acute respiratory distress syndrome? Ann Transl Med 2019;7(19):510. doi: 10.21037/atm.2019.08.25
Nutrients 2016;8:76.

60. Holick MF. Vitamin D deficiency. N Engl J Med 2007;357:266-81.

61. Parekh D, Thickett DR, Turner AM. Vitamin D deficiency and acute lung injury. Inflamm Allergy Drug Targets 2013;12:253-61.

62. Ponnarmeni S, Angurana SK, Singhi S, et al. Vitamin D deficiency in critically ill children with sepsis. Paediatr Int Child Health 2016;36:15-21.

63. Linko R, Karlsson S, Pettilä V, et al. Serum zinc in critically ill adult patients with acute respiratory failure. Acta Anaesthesiol Scand 2011;55:615-21.

64. Vahdat Shariatpanahi Z, Mokhtari M, Taleban FA, et al. Effect of enteral feeding with ginger extract in acute respiratory distress syndrome. J Crit Care 2013;28:217.e1-6.

65. Berger MM, Chioléro RL. Antioxidant supplementation in sepsis and systemic inflammatory response syndrome. Crit Care Med 2007;35:S584. 